\title{
Tetrad in Curved Space-Time in Cosmological General Theory
}

\section{of Relativity}

\author{
Sangwha-Yi* \\ Department of Math, Taejon University 300-716, South Korea
}

*Corresponding Author: Sangwha-Yi, Department of Math, Taejon University 300-716, South Korea

Abstract: In the cosmological general theory of relativity, we define the tetrad that moves in r-axis in the curved space-time. We study an accelerated motion in curved space-time.

Keywords: Cosmological General Theory of Relativity; Tetrad in Curved Space-Time; Constant Accelerated Motion

PACS Number: 04,04.90.+e

\section{INTRODUCTION}

This theory's aim is to define tetrad that moves in r-axis in the curved space-time.

Schwarzschild solution is

$$
d \tau^{2}=\left(1-\frac{2 G M}{r c^{2}}\right) d t^{2}-\frac{1}{c^{2}}\left[\frac{d r^{2}}{1-\frac{2 G M}{r c^{2}}}+r^{2} d \theta^{2}+r^{2} \sin ^{2} \theta d \phi^{2}\right]
$$

In this case, the cosmological time $t_{0}$ is the present cosmological time for constant accelerated motion in cosmological general theory of relativity [2,3]. The ratio of the universe's expansion is

$$
\Omega\left(t_{0}\right)=1
$$

Hence, in this time, the cosmological general theory of relativity and the cosmological special theory of relativity do the general relativity theory and the special relativity theory.

In this time, a moving matter's acceleration is the constant acceleration $a_{0}$ in the Schwarzschild space-time.

$$
\begin{aligned}
& a_{0}=\frac{d}{d t}\left(\frac{u}{\sqrt{1-\frac{2 G M}{r c^{2}}-\frac{u^{2}}{c^{2}}}}\right) \\
& a_{0} t=\frac{u}{\sqrt{1-\frac{2 G M}{r c^{2}}-\frac{u^{2}}{c^{2}}}}, \quad u=\sqrt{1-\frac{2 G M}{r c^{2}}} \frac{a_{0} t}{\sqrt{1+\frac{a_{0}^{2} t^{2}}{c^{2}}}}
\end{aligned}
$$

If $\frac{d \theta}{d t}=\frac{d \phi}{d t}=0$, the solution is 


$$
d \tau^{2}=\left(1-\frac{2 G M}{r c^{2}}\right) d t^{2}-\frac{1}{c^{2}} \frac{d r^{2}}{1-\frac{2 G M}{r c^{2}}}
$$

In this time, if we use $\psi$,

$$
\begin{aligned}
& 1=\left(1-\frac{2 G M}{r c^{2}}\right)\left(\frac{d t}{d \tau}\right)^{2}-\frac{1}{c^{2}} \frac{1}{1-\frac{2 G M}{r c^{2}}}\left(\frac{d r}{d \tau}\right)^{2} \\
& \cosh \psi=\sqrt{1-\frac{2 G M}{r c^{2}}} \frac{d t}{d \tau}, \quad \sinh \psi=\frac{1}{c} \frac{1}{\sqrt{1-\frac{2 G M}{r c^{2}}}} \frac{d r}{d \tau}
\end{aligned}
$$

Therefore, r-axis's velocity $V_{r}$ is

$$
V_{r}=\frac{1}{\sqrt{1-\frac{2 G M}{r c^{2}}}} \frac{d r}{d t}=u=\sqrt{1-\frac{2 G M}{r c^{2}}} \frac{a_{0} t}{\sqrt{1+\frac{a_{0}{ }^{2} t^{2}}{c^{2}}}}
$$

According to $\mathrm{Eq}(5), \mathrm{Eq}(6)$,

$$
\begin{aligned}
& \frac{1}{c} \frac{d r}{\sqrt{1-\frac{2 G M}{r c^{2}}}}=\frac{1}{c} \frac{a_{0} t}{\sqrt{1+\frac{a_{0}^{2} t^{2}}{c^{2}}}} \sqrt{1-\frac{2 G M}{r c^{2}}} d t, \cosh \psi=\sqrt{1-\frac{2 G M}{r c^{2}}} \frac{d t}{d \tau} \\
& =\frac{1}{c} \frac{a_{0} t}{\sqrt{1+\frac{a_{0}^{2} t^{2}}{c^{2}}}} \cosh \psi d \tau=\sinh \psi d \tau \\
& \frac{1}{\cosh ^{2} \psi}=1-\left(\frac{\sinh \psi}{\cosh \psi}\right)^{2}=1-\left(\frac{a_{0} t / c}{\sqrt{1+\frac{a_{0}^{2} t^{2}}{c^{2}}}}\right)^{2}=\frac{1}{1+\frac{a_{0}^{2} t^{2}}{c^{2}}}
\end{aligned}
$$

Hence,

$\cosh \psi=\sqrt{1+\frac{a_{0}{ }^{2} t^{2}}{c^{2}}} \quad, \quad \sinh \psi=\frac{a_{0} t}{c}$

$\cosh \psi=\sqrt{1-\frac{2 G M}{r c^{2}}} \frac{d t}{d \tau}=\sqrt{1+\frac{a_{0}^{2} t^{2}}{c^{2}}}, \sinh \psi=\frac{1}{c} \frac{1}{\sqrt{1-\frac{2 G M}{r c^{2}}}} \frac{d r}{d \tau}=\frac{a_{0} t}{c}$

Therefore, 
$\frac{d t}{d \tau}=\frac{\sqrt{1+\frac{a_{0}^{2} t^{2}}{c^{2}}}}{\sqrt{1-\frac{2 G M}{r c^{2}}}} \quad, \quad \frac{1}{c} \frac{d r}{d \tau}=\frac{a_{0} t}{c} \sqrt{1-\frac{2 G M}{r c^{2}}}$

\section{Tetrad in Curved SPaCe-Time}

The tetrad $e_{a}^{\mu}$ is the unit vector that is each other orthographic.

$e_{a}{ }^{\mu} e_{b}{ }^{v} g_{\mu v}=\eta_{a b}$

Therefore, $\mathrm{Eq}(11)$ is

$$
\begin{aligned}
& g_{\mu \nu} e_{0}{ }^{\mu}(r, t) e_{0}{ }^{\nu}(r, t)=\eta_{00}=-1 \\
& d \tau^{2}=-\frac{1}{c^{2}} g_{\mu \nu} d x^{\mu} d x^{\nu} \\
& \rightarrow-1=g_{\mu \nu}\left(\frac{1}{C} \frac{d x^{\mu}}{d \tau}\right)\left(\frac{1}{C} \frac{d x^{\nu}}{d \tau}\right)=g_{\mu \nu} e_{0}^{\mu}(r, t) e_{0}{ }^{\nu}(r, t)
\end{aligned}
$$

According to $\mathrm{Eq}(10), \mathrm{Eq}(12)$

$$
\begin{aligned}
& e_{0}^{\alpha}(r, t)=\frac{1}{c} \frac{d x^{\alpha}}{d \tau} \\
& =\left(\frac{\sqrt{1+\frac{a_{0}^{2} t^{2}}{c^{2}}}}{\sqrt{1-\frac{2 G M}{r c^{2}}}}, \frac{a_{0} t}{c} \sqrt{1-\frac{2 G M}{r c^{2}}}, 0,0\right)
\end{aligned}
$$

About $\theta$-axis's and $\phi$-axis's orientation

$$
\begin{aligned}
& g_{22} e_{2}^{2}(r, t) e_{2}^{2}(r, t)=\eta_{22}=1, \quad e_{2}^{\alpha}(r, t)=\left(0,0, \frac{1}{r}, 0\right) \\
& g_{33} e_{3}^{3}(r, t) e_{3}^{3}(r, t)=\eta_{33}=1, \quad e_{3}^{\alpha}(r, t)=(0,0,1 / r \sin \theta, 0)
\end{aligned}
$$

And the other vector $e_{1}^{\alpha}(r, t)$ has to satisfy the tetrad condition, Eq (11)

$$
\begin{aligned}
& g_{00} e_{0}^{0}(r, t) e_{1}^{0}(r, t)+g_{11} e_{0}{ }^{1}(r, t) e_{1}^{1}(r, t)=\eta_{01}=0 \\
& e_{1}^{\alpha}(r, t)=\left(\frac{a_{0} t / c}{\sqrt{1-\frac{2 G M}{r c^{2}}}}, \sqrt{1+\frac{a_{0}^{2} t^{2}}{c^{2}}} \sqrt{1-\frac{2 G M}{r c^{2}}}, 0,0\right)
\end{aligned}
$$

\section{CONCLUSiON}

In the cosmological general theory of relativity, we define the tetrad that moves in r-axis in the curved space-time. 


\section{REFERENCES}

[1]S.Yi,"Curvature Tensor of the Stationary Accelerated Frame in Gravity Field", African Review of Physics,9,59,(2014)

[2]S.Yi,"Cosmological Special Theory of Relativity”, International Journal of Advanced Research in Physical Science, 7,11,(2020),pp 4-9

[3]S.Yi,"Cosmological Gpecial Theory of Relativity", International Journal of Advanced Research in Physical Science,8,2,(2021),pp 22-26

[4]S.Weinberg, Gravitation and Cosmology (John wiley \& Sons,Inc,1972)

[5]P.Bergman, Introduction to the Theory of Relativity (Dover Pub. Co.,Inc., New York,1976), Chapter V

[6]C.Misner, K,Thorne and J. Wheeler, Gravitation(W.H.Freedman \& Co.,1973)

[7]S.Hawking and G. Ellis, The Large Scale Structure of Space-Time(Cam-bridge University Press,1973)

[8]R.Adler, M.Bazin and M.Schiffer, Introduction to General Relativity(McGraw-Hill,Inc., 1965)

[9]M.Schwarzschild, Structure and Evolution of the Stars(Princeton University Press, 1958; reprint,Dover,N.Y.1965), chapter II

[10]S.Chandrasekhar, Mon,Not.Roy.Astron.Soc.95.207(1935)

[11]C.Rhoades, "Investigations in the Physics of Neutron Stars", doctoral dissertation, Princeton University [12]J.Oppenheimer and H.Snyder, phys.Rev.56,455(1939)

Citation: Sangwha-Yi (2021). Tetrad in Curved Space-Time in Cosmological General Theory of Relativity. International Journal of Advanced Research in Physical Science (IJARPS) 8(4), pp.16-19, 2021.

Copyright: () 2021 Authors, This is an open-access article distributed under the terms of the Creative Commons Attribution License, which permits unrestricted use, distribution, and reproduction in any medium, provided the original author and source are credited. 